\title{
Recent Advances in Social \& Cognitive Robotics and Imminent Ethical Challenges
}

\author{
Ali Meghdari ${ }^{1}$, Minoo Alemi ${ }^{1,2}$

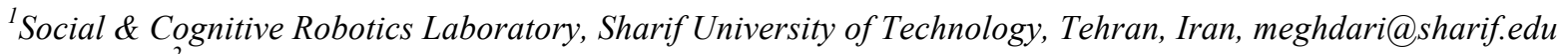 \\ ${ }^{2}$ Department of Humanities, Islamic Azad University-West Tehran Branch, Tehran, Iran
}

\begin{abstract}
One of the main challenges in developing and applying modern technologies in our societies is the identification and consideration of ethical issues. With the dramatic growth of emerging technologies in today's societies, such as social robots, lifelike computer graphics (avatars), and virtual reality tools and haptic systems, the social complexity of these challenges is on the rise. To be prepared to face these challenges, researchers in engineering sciences and humanities are forming interdisciplinary research groups/activities and try to determine the rate of evolution as well as the effects of socio-cognitive systems on human interaction with intelligent tools and/or artificial agents. A growing number of social robotics researchers are seeking to create a framework to benefit from humanities, philosophy, sociology, and social neuroscience expertise and research. Likewise, growing trends of mutual collaboration among scholars in the field of human sciences, linguistics, and psychology with the robotics scientists are producing quite noticeable valuable results. This paper presents an overview of the novel and multidisciplinary area of socio-cognitive robotics, and further explores the possible ethical challenges of emerging technologies on education, culture, entertainment, gaming, nursing, and therapy. In addition, some key ethical features based on our past and present research experiences in a variety of areas in designing safe social robots are also presented.
\end{abstract}

KEYWORDS: cognitive robotics, emerging technologies, ethical issues, human robot interaction, roboethics, robot-rights, social robots

\section{Introduction}

The emergence of robotics technology is developing much quicker than previously thought and/or anticipated such that robots in society will soon be as ubiquitous as computers are today. Society has long been concerned with the impact of robotics technology from nearly a century ago ( $\mathrm{C}^{`}$ apek, 1921) when the word "Robot" was devised for the first time. Modern literature about robots features cautionary accounts about insufficient programming, evolving behavior, errors, and other issues that make robots unpredictable and potentially risky or dangerous (Asimov, 1942-1985).

In recent years, with the advancement of technology, social robots have found broader applications in the private and public sectors, such as educational and cultural affairs (Alemi, Meghdari et al. 2014-2017), games and entertainment, clinical and rehabilitation, nursing of children and/or elderly, search and rescue operations, and so on. For example, social robots such as ASIMO, $\mathrm{Nao}$, iCub, ARASH, and RASA in research and education have been developed for "Edutainment" or "education-entertainment" purposes. Though they may lack a clear use, such as serving specific manufacturing functions, they aid researchers in the study of cognition (both human and artificial), motion, and other areas related to the advancement of robotics serving our society. In addition, a few medical and healthcare toy-like robots, such as PARO, which looks like a baby seal, or ARASH (Meghdari, Shariati, and Alemi 2018), which is a humanoid, have been designed for therapeutic purposes such as reducing distress, stimulating cognitive activity, teaching specific subjects, and improving socialization. Similarly, Sharif University of Technology's socially assistive robot RASA (Meghdari, Alemi, and Zakipour 2018) has been developed to help coach and teach Persian SignLanguage to Iranian deaf children. Personal care and companion robots are increasingly being used to care for the elderly and children, such as RI-MAN, PaPeRo, and CareBot. Surprisingly, although not too hard to imagine, relationships of a more intimate nature have not quite been satisfied by robots yet (Veruggio 2005).

To enhance the ability of social robots to successfully operate in such roles and environments, it is necessary to upgrade them to a new level of physical skills and cognitive capabilities (Meghdari et al. 2013-2018). In the design and construction of social robots the consideration of ethical concerns, in 
addition to physical challenges associated with the needs and expectations of the human mind, is of utmost important to researchers in this multidisciplinary field (Lin, Abney, and Bekey 2012). Humanrobot (a machine with a higher physical and social ability) interactions (Saffari et al. 2015), are somewhat different compared to other types of human-machine interactions (i.e. with a computer, cell phone, or other smart device). Therefore, it is essential for researchers, scholars, and users to clearly identify, understand, and consider these differences and ethical challenges so that they can benefit from the assistance of social robots as a powerful tool in providing modern and quality services to society (Taheri et al. 2014-2018). In this paper, the novel and multidisciplinary area of sociocognitive robotics, and the ethical challenges of emerging technologies are explored in in education, culture, entertainment, gaming, nursing, and therapy (Ghorbandaei Pour et al. 2018). In addition, key ethical features based on our past and present research experiences in a variety of areas in designing safe social robots will be presented.

\section{Social-Cognitive Robotics}

Robotics technology in recent years has extended its applications from factories to more generalpurpose practices in society such as the use of robots in clinical and rehabilitation, nursing and elderly care, search and rescue operations, etc. However, for robotics technology to be successful in such environments it is necessary for robots to gain a new level of strength, robustness, physical skills, and improved cognitive ability and intelligence. The design and construction of Social Robots (SR) faces many challenges, one of the most important is to build robots that can comply with the needs and expectations of the human mind with cognitive capabilities. It is clear that the way we communicate with machines with a higher quality physical and divine appearance would certainly differ from the way we interact with a computer, cell phone, or other smart devices. Therefore, Social-Cognitive Robotics (SCR) is a transdisciplinary area of research and a basis for the human-centered design of technology-oriented systems to improve human knowledge functions, judgements and decision making, collaborations, and learning. It draws on the knowledge of prospective users and involves them in the design process. It goes beyond distinct users to examine the activity schemes of people and their interaction with technology, including their social interactions, styles and working strategies, language and communication patterns to form a combination of human knowledge and activity that can be used to update system design.

The SCR framework consists of two main areas: one being action analysis to interpret how people work and interact with their current tools and technologies, and the other being the systems design to build and implement new interactive technologies. Social-cognitive robotics has been evolving and verified through a series of projects to develop advanced and modern technology-based systems to support learnings and knowledge functions, and is beginning to play an effective role in societies across the globe. SCR or Socio-Cognitive Robotics is the interdisciplinary study and application of robots that are able to teach, learn and reason about how to behave in a complex world. They are robots that can interact and communicate among themselves, with humans and the environment within the cognitive, cultural, linguistics, psychological, social, and therapeutic framework attached to their role.

In recent years, we have designed and constructed several social robots as clinical and educational assistants for social interventions, treatment, and education of children with disabilities like autism, down syndrome, cancer distress, hearing impairment, etc. (Meghdari, Alemi, Shariati, Zakipour 2015-2018). Figures 1 to 5 shows sample applications of social robots providing educational, therapy, and entertainment services to healthy children as well as children with hearing impairments, children with cancer, children with autism spectrum disorder, and children with downsyndrome in the presence of human psychologists and operators, respectively. Initial investigations clearly indicate that social robots can play a positive role in the improvement of children's social performance, reduction of distress during treatments, and enhancing their learning abilities. 

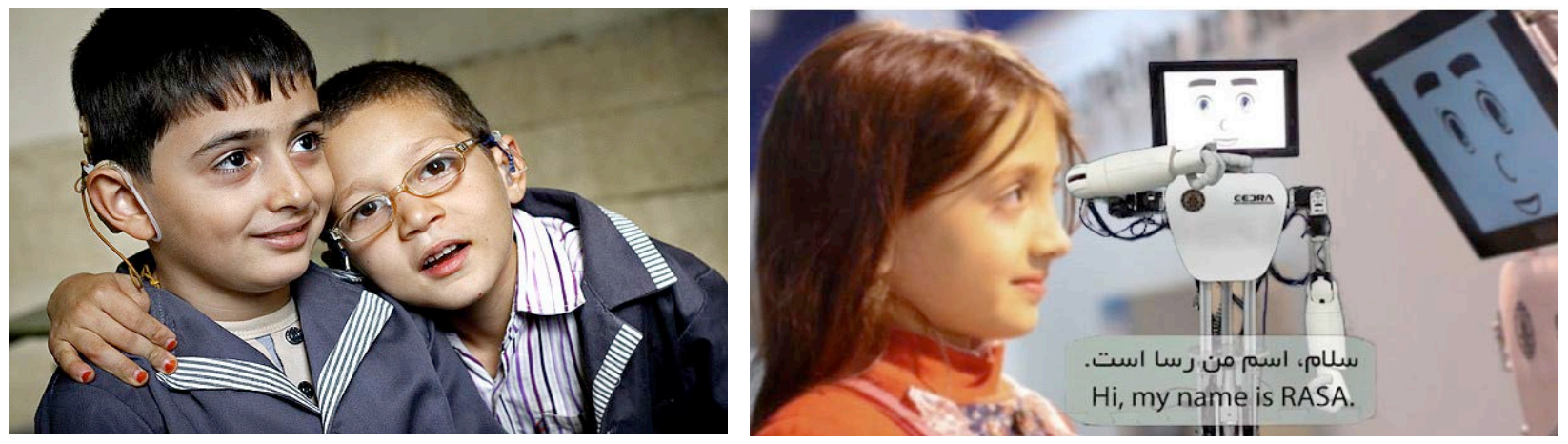

Figure 1. RASA: A social robot for teaching sign-language to deaf children

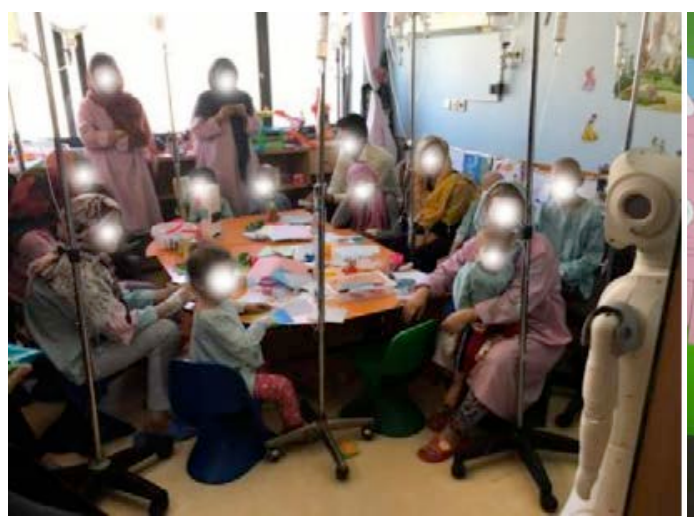

(ARASH)

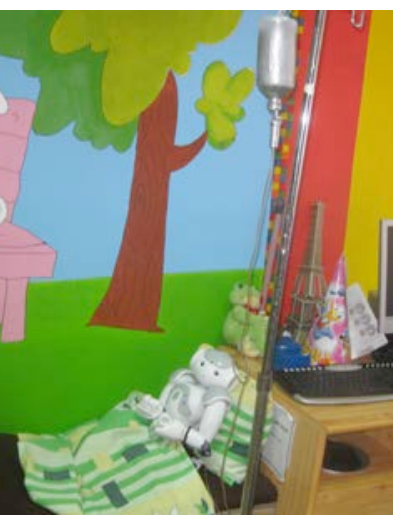

(NAO)

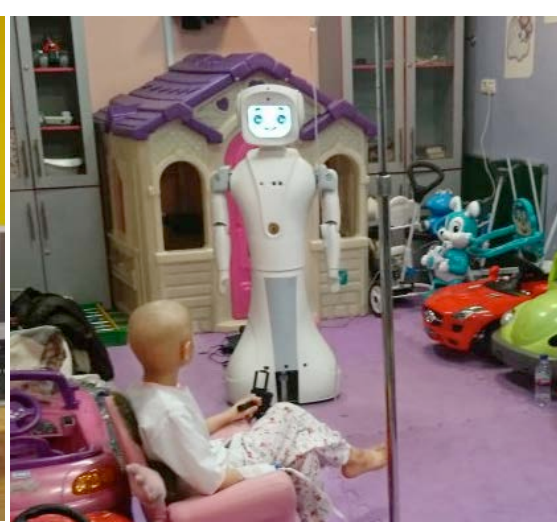

(ARASH)

Figure 2. ARASH: A social robot companion for kids with cancer in a pediatric hospitals environment
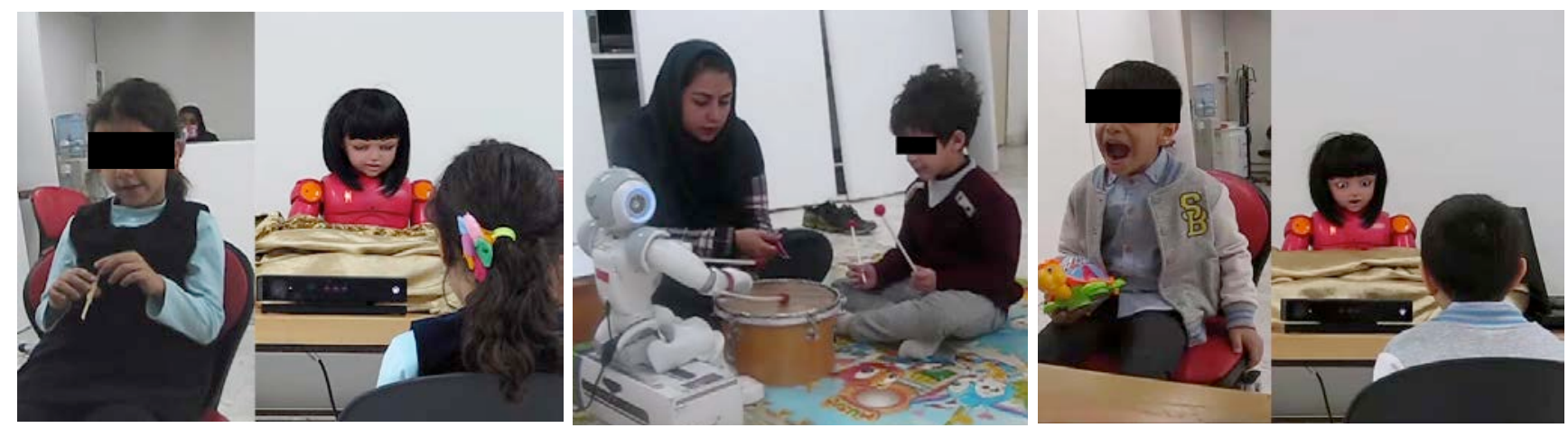

Figure 3. NAO \& Alice: Social robots for treatment of kids with Autism Spectrum Disorder (ASD)
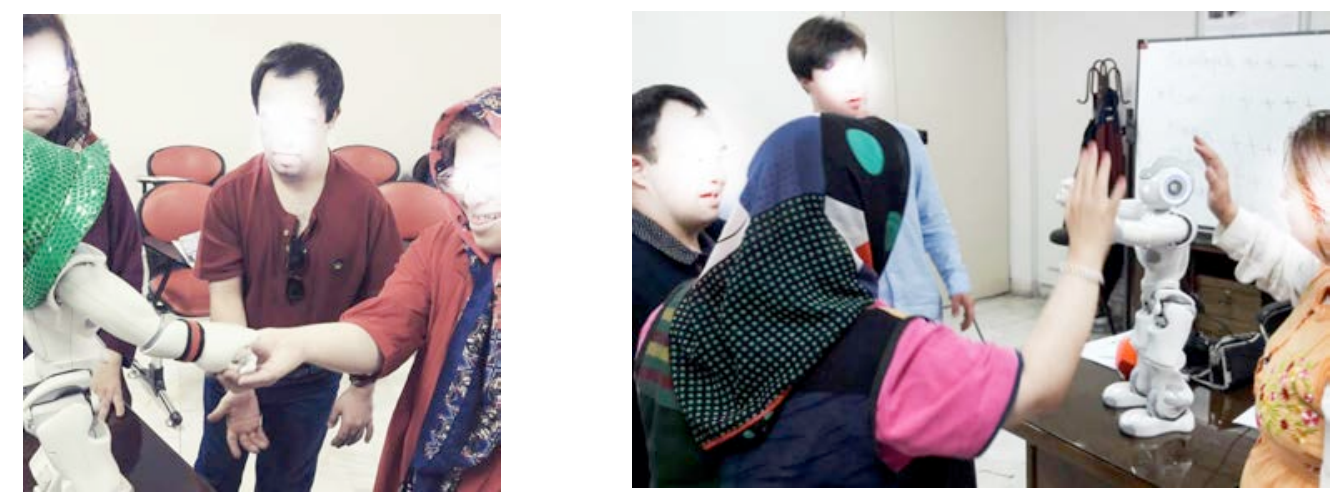

Figure 4. English vocabulary learning by Iranian EFL students with Down Syndrome using the NAO social robot 

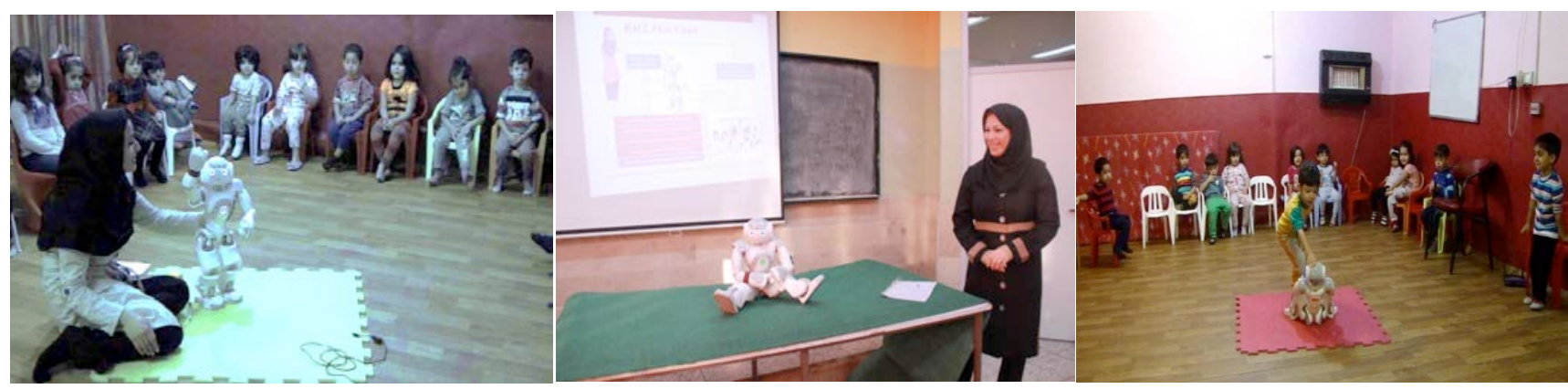

Figure 5. Robot Assisted Language Learning (RALL) for Iranian EFL students using NAO

\section{Ethics and Morality - Social Robots}

Social robotics technology promises a host of benefits that are fascinating and ingenious, but, as with other emerging areas of science, they also come with challenges and new queries that society must be ready to confront. This is not unexpected, considering the previous disruptive evidence observed in the nature of other technologies. As a result the term "RoboEthics" has recently gained considerable attention among humanities and robotics engineers. One may ask: if "robotics and ethics" is a new science or an integral part of Engineering? Robotics is actually a discipline born from computer science, artificial intelligence, mechanics, physics, math, electronics, automation, and control and cybernetics.

Additionally, social and cognitive robotics draws on many other disciplines like logic, neuroscience, biology, linguistics, philosophy, natural history and arts. What specifies emergence of socio-cognitive robotics is that humanity is at the threshold of replicating an intelligent and autonomous agent. In other words, robotics de facto unifies two cultures, engineering science and humanities, and the primary precondition for roboethics is the unity of these cultures. In this context, complex concepts (like learning, perception, decision-making, freedom, judgement, emotions, etc.) may not have the same semantic meaning for humans and machines. Here we plan to state and categorize the numerous possible interrelated areas of ethical and social concerns facing social robotics and provide some typical questions.

First, let us briefly review the words "Morality" and "Ethics". Morality governs principles concerning the distinction between right and wrong or good and bad behavior, whereas Ethics is moral principles that govern a person's or group's behavior. With this in mind, as ethics is the science of morals, robotics is the science of robots.

In 1950, Isaac Asimov introduced the idea of good robots (Androids) in his stories and popularized the word "Robotics". He was the one who first introduced the first ethical laws in robotics. They are:

- $\underline{1}^{\text {st } L a w}$ : A robot may not injure a human being or, through inaction, allow a human being to come to harm.

- $2^{\text {nd }} L a w$ : A robot must obey the orders given it by human beings except where such orders conflict with the first law.

- $\underline{3}^{\text {rd } L a w}$ : A robot must protect its own existence as long as such protection does not conflict with the first or second law.

With this background, the discussion should analyze the effects of robotics in general and social robots in particular in our societies, from the declared principles considering both cultural and religious variations, in the applications fields where the potential complications are more significant and apparent. Some of the main principles to be followed are as follows: human rights/dignity, equality and justice, benefits and damage, cultural diversity and pluralism, religious variety, nondiscriminating, independence and individual accountability, privacy and confidentiality, unity and collaboration, social responsibility, benefits sharing, environmental obligation, etc. 
- Economy and Employment: advancement of technologies frequently disrupts employment patterns. Robots and social machines have already replaced people in a variety of jobs (e.g. airports smart flight check-in kiosks). This disruption will only increase as machines become more intelligent.

- Impacts on Society: these smart machines and/or social robots will definitely influence our life when they act as our servants, housekeepers, babysitters, and drivers. Our lives could soon become dependent on them. Like the social networking and email capabilities of the internet, social robotics may overwhelmingly impact our relationships. Already, social robots are beginning to take care of our elderly and children, and some studies are currently underway on the effects of such care (Alemi, Meghdari, et al., 2014-2018).

- Medical and Health Care: the health ethics of bio-robotics technology can be concerned with diagnosing health problems, progress of surgery, etc.

- Affordability and Ease of Access: robotics researchers and developers should create social robots and intelligent machines commercially affordable and readily available to serve all humanity.

- $\quad$ Misuse and Violence: just like many other technologies, robots have the potential to be used in wars, violence, terrorism, etc. with an intense effect.

Errors and Safety: The main and leading concern about any new and emerging technology is to be safe and error free. Therefore, sufficient and numerus tests on health and safety must be performed by developers and/or well-known independent sources before applying any technology into the marketplace and society. In robotics, the safety issue has to do with their software and/or hardware designs. Even a tiny software flaw or a manufacturing defect in an intelligent machine, like a smart car or a social robot, could lead to fatal results.

Morals, Ethics, and the Law: As social robots become more intelligent and autonomous and exhibit enough of the features that typically define an individual person, it may be conceivable to assign them responsibility and use them in social, educational, and therapeutic settings. If this seems too implausible, consider that there is ongoing work in integrating computers and robotics with biological brains (Warwick and Shah, 2014). A cognizant human brain (and its physical body) apparently has human-rights; hence, replacing parts of the brain with artificial ones, while not harming its function, would seem to preserve those rights. As seen in Figure 6, at some point in the future we may face a situation in which more than half of the brain or body is artificial, making the organism more robotic than human, which makes the issue of robot-rights more reasonable.
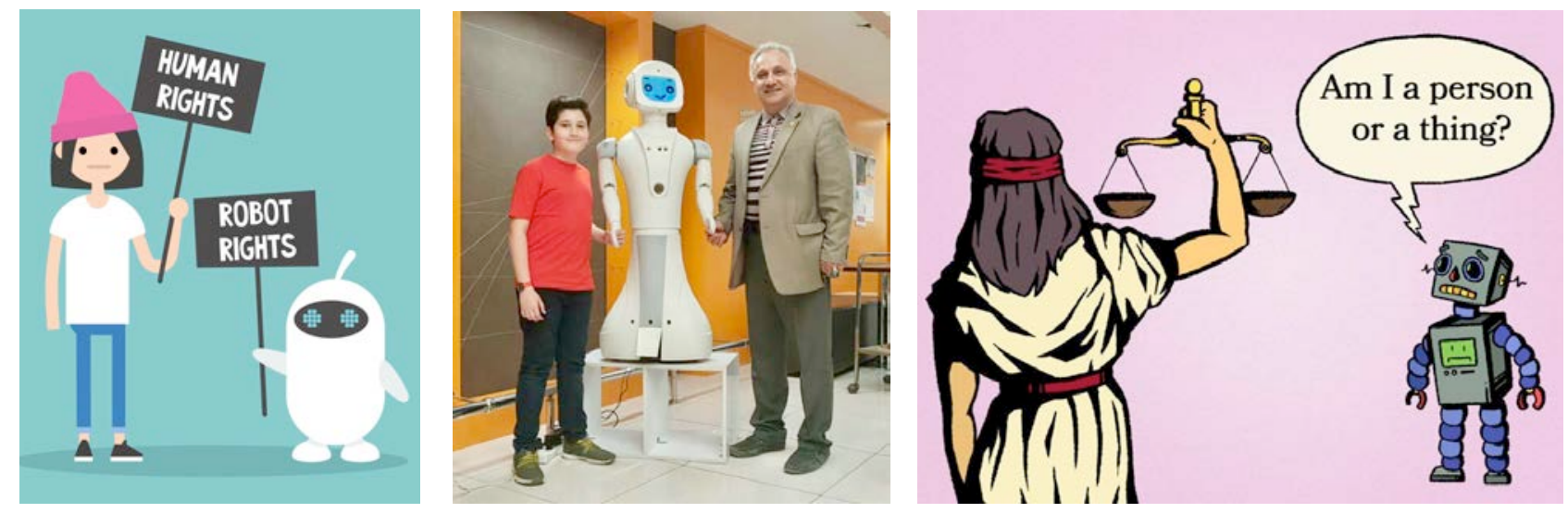

Figure 6. Robot rights vs. Human rights.

With regard to the risk of robotic malfunctions and errors, it may not be clear who is accountable for possible subsequent damage. Product legal responsibility laws are mostly untested in robotics; but nevertheless, continue to evolve in a way that releases manufacturers from obligation, as 
it generally occurs through end-user license contracts. A usual way to minimize the risk of damage from social robots is to program them to obey predefined regulations or follow a code-of-ethics. Of course, this is much easier said than done. Ethical laws can be vague and change depending on circumstances social robots may not currently be refined enough to recognize and follow. Even the laws of robotics in Asimov's stories, as sophisticated and adequate as they appear to be, can create gaps that result in some form of impairment (Asimov, 1942-1985).

Additional and significant areas in ethical laws are confidentiality and privacy. Several issues and reasons cause these to be of main concern. This includes the appearance of small size digital cameras, miniature recording devices, advanced sensors, as well as a growing emphasis on security at the expense of privacy (e.g., the large number of surveillance cameras in some cities to monitor traffic and/or prevent crimes). Current and future police social robots could conduct intimate shadowing at a distance, to detect hidden drugs or weapons and identify faces discreetly; once linked to databases, they could also run background checks on an individual's criminal, driving, medical, banking, or other records to determine if the person should be detained. Some of the questions that may be raised here are:

- What ethical theory shall be used to program code-of-ethics in controlling the robot's action?

- Are there any lawful/ethical fears in designing robots that can independently harm people?

- Should robots simply be considered as tools, like weapons/computers, and regulated similarly?

- Is it morally allowable to assign obligation for our kids and elderly to robots as a substitute for human companionship?

- is it morally right to use robots as companions for other purposes such as pets, entertainment, or an intimate relationship?

- When can we treat a social robot as a "person or human" hence giving it some rights and duties, and if that point is reached should we liberate our social robot instead of treating them like possessions or "slaves"?

- Are there specific moral reservations over placing social robots in positions of authority, such as police, security guards, teachers, or any other government roles or offices, in which humans would be expected to obey them?

\section{Conclusions}

Social and cognitive robotics is rapidly becoming one of the leading fields of science and technology involving a deep level of human-machine interaction. We can predict that humanity will soon coexist with the first man-made intelligence machines we have ever come into contact with - smart social robots. One may conclude that roboethics entails the ethical norms built into social robots, the ethics of design and production of various purpose robots, and the ethics of handling and application of robots. This will be an event rich in challenging ethical, social, and economic issues. As a group of researchers and educators in the emerging field of robotics, we hope that this paper on social robot ethics will provide and motivate (in view of cultural, religious, and ethical differences) greater discussions to tackle many of these issues - in and outside the classroom - across the broad range of topics, as briefly described here. At the same time, one should also recognize the fact that these emerging technologies seem to jump out of the pages of science fiction literature, and the ethical dilemmas they raise also seem too distant to consider, if not altogether unreal. But as Isaac Asimov predicted: "It is change, continuing change, inevitable change, that is the dominant factor in society today. No sensible decision can be made any longer without taking into account not only the world as it is, but the world as it will be. . . This, in turn, means that our statesmen, our businessmen, our everyman must take on a science fictional way of thinking" (Asimov, 1978). With human ingenuity, what was once fiction is becoming fact, and the new challenges it brings are all too real to ignore.

\section{Acknowledgement}

This work is supported in part by the Iranian National Science Foundation (INSF, http://en.insf.org/) to promote Social and Cognitive Robotics Chair at Sharif University of Technology, Tehran, Iran. 


\section{References}

Alemi, M., Meghdari, A., Ghanbarzadeh Ash., Jafari Moghaddam, L., Ghanbarzadeh, A. 2014. "Impact of a Social Humanoid Robot as a Therapy Assistant in Children Cancer Treatment", Proc. of the 6th Int. Conf. on Social Robotics (ICSR), Springer, Oct. 2014, Sydney, Australia.

Alemi, M. Meghdari, A., Mahboub Basiri, N., Taheri, A.R. 2015. "The Effect of Applying Humanoid Robots as Teacher Assistants to Help Iranian Autistic Pupils Learn English as a Foreign Language." Lecture Notes in Computer Science (LNCS): Social Robotics 9388: 1-10, Springer, Oct. 2015.

Alemi, M. Ghanbarzadeh, A., Meghdari, A. "Clinical Application of a Humanoid Robot in Pediatric Cancer Interventions.” Int. Journal of Social Robotics (IJSR) 8 (5): 743-759, Springer, 2016.

Alemi, M., Meghdari, A., Haeri, N.S. 2017. "Young EFL Learners' Attitude Towards RALL: An Observational Study Focusing on Motivation, Anxiety, and Interaction." Lecture Notes in Computer Science (LNCS): Social Robotics 10652: 252-261, Springer, Nov. 2017.

Alemi, M., Meghdari, A., Saffari, E. 2017. "RoMa: A Hi-tech Robotic Mannequin for the Fashion Industry." Lecture Notes in Computer Science (LNCS): Social Robotics 10652: 209-219, Springer, Nov. 2017.

Asimov, I. 1942. Runaround, In I, Robot, 33 - 51 . London: Grafton Books.

Asimov, I. 1950. I, Robot (2004 ed.). New York : Bantam Dell.

Asimov, I. 1978. My own view. In The Encyclopedia of Science Fiction, ed. Robert Holdstock, N.Y.: St. Martin's Press.

Asimov, I. 1985. Robots and Empire. New York: Doubleday.

C`apek, K. 1921. Rossum's Universal Robots (2004 ed.), trans. Claudia Novack. New York: Penguin Group .

Ghorbandaei Pour, A., Taheri, A.R., Alemi, M., Meghdari, A. 2018. "Human-Robot Facial Expression Reciprocal Interaction Platform: Case Studies on Children with Autism." Int. Journal of Social Robotics 10 (2): 179-198.

Lin, P., Abney, K., Bekey, G.A. 2012. ROBOT ETHICS: The Ethical and Social Implications of Robotics. London, England: The MIT Press.

Meghdari, A., Alemi, M., Ghazisaedy, M., et. al. 2013. "Applying Robots as Teaching Assistant in EFL Classes at Iranian Middle-Schools", Proc. of the Int. Conf. on Education and Modern Educational Technologies, Sept. 2013, Venice, Italy.

Meghdari, A., Alemi, M., Ghazisaedy, M. 2014. "The Effect of Employing Humanoid Robots for Teaching English on Students' Anxiety and Attitude", Proc. of the 2nd RSI Int. Conf. on Robotics and Mechatronics (ICRoM), Oct. 1517, 2014, IEEE, Tehran, Iran.

Meghdari, A., Alemi, M., Ghazisaedy, M. 2014. "Employing Humanoid Robots for Teaching English Language in Iranian Junior High-Schools.” Int. Journal of Humanoid Robotics 11(3), September 2014.

Meghdari, A., Alemi, M., Ghazisaedy, M. 2015. “The Impact of Social Robotics on L2 Learners' Anxiety and Attitude in English Vocabulary Acquisition.” Int. Journal of Social Robotics 7(4): 523-535, August 2015.

Meghdari, A., Alemi, M. 2015. "Socio-Cognitive Robotics: Mysteries and Needs." Proc. of 1st Int. and 4th National ISEE Conference on Engineering Education, November 10-11, 2015, Shiraz, Iran.

Meghdari, A., Alemi, M. 2016. "Socio-Cognitive Robotics: Mysteries and Needs." Iranian Journal of Engineering Education; Iranian Academy of Sciences 18(9), Spring 2016.

Meghdari, A., Alemi, M., Zakipour, M., Kashanian, S.A. 2018. "Design and Realization of a Sign Language Educational Humanoid Robot.” Journal of Intelligent \& Robotic Systems, pp. 1-15, Springer, 2018.

Meghdari, A., Alemi, M., Ghorbandaei Pour, A., Taheri, A.R. 2016. "Spontaneous Human-Robot Emotional Interaction through Facial Expressions.” LNCS: Social Robotics 9979: 351-361, Springer, 2016.

Meghdari, A., Shariati, A., Alemi, M., Vossoughi, G.R., et. al. 2018. “Arash: A Social Robot Buddy to Support Children with Cancer in a Hospital Environment." Proc. of the IMechE, Part H: Journal of Engineering in Medicine 232(6): 605-618.

Meghdari, A., Alemi, M., Taheri, A.R., Hatefipour, M. 2016. “The Social WATERobot: An Exciting Educational Tool for Teaching Children about Water Awareness and Conservation." Proc. Int. Conf. on Water and Environment in the new Millennium: Education \& Capacity Building (WENM2016), pp. 295-298, Dec. 3-5, 2016, University of Tehran, Iran.

Meghdari, A., Bagheri Shouraki, S., Siyamy, A., Shariati, A. 2016. "The Real-Time Facial Imitation by a Social Humanoid Robot", Proc. of the 4th RSI Int. Conf. on Robotics and Mechatronics (ICRoM), October 2016, IEEE, Tehran, Iran. DOI: 10.1109/ICRoM.2016.7886797.

Saffari, E., Meghdari, A., Vazirnezhad, B., Alemi, M. 2015. “Ava (A Social Robot): Design and Performance of a Robotic Hearing Apparatus.” LNCS: Social Robotics 9388: 440-450, Springer, Oct. 2015.

Taheri, A.R., Alemi, M., Meghdari, A., Pouretemad, H.R., Mahboob Basiri, N. 2014. "Social Robots as Assistants for Autism Therapy in Iran: Research in Progress." Proc. of the 2nd RSI Int. Conf. on Robotics and Mechatronics (ICRoM), Oct. 15-17, 2014, IEEE, Tehran, Iran.

Taheri, A.R.. Alemi, M., Meghdari, A., Pouretemad, H.R., et. al. 2015. "Impact of Humanoid Social Robots on Treatment of a Pair of Iranian Autistic Twins." Lecture Notes in Computer Science (LNCS): Social Robotics 9388: 623-632, Springer, Oct. 2015.

Taheri, A.R., Meghdari, A., Alemi, M., Pouretemad, H.R., et. al. 2016. "Social Robots and Teaching Music to Autistic Children: Myth or Reality?” LNCS: Social Robotics 9979: 541-550, Springer, 2016. 
Taheri, A.R., Meghdari, A., Alemi, M., Pouretemad, H.R., et. al. 2018. "Human-Robot Interaction in Autism Treatment: A Case Study on Three Pairs of Autistic Children as Twins, Siblings, and Classmates." Int. Journal of Social Robotics 10(1): 93-113.

Veruggio, G. 2005. "The Birth of Roboethics." ICRA 2005, IEEE Int. Conference on Robotics and Automation: Workshop on Robo-Ethics, Barcelona, April 18, 2005.

Warwick, K., Shah, H., et. al. 2014. "How Good Robots Will Enhance Human Life." In book: Treatise on Good Robots Edition: PRAXIOLOGY: The International Annual of Practical Philosophy and Methodology Vol. 21, Book Chapter, Transaction Publishers, USA Editors: Krzysztof Tchoń, Wojciech W. Gasparski, January 2014. 